8. Nine new cases of COVID-19 infection confirmed. Singapore Ministry of Health website. https://www.moh.gov.sg/news-highlights/details/ninenew-cases-of-covid-19-infection-confirmed. Published March 12, 2020. Accessed April 3, 2020.

9. Boorstein M. The Georgetown church quarantined by DC's coronavirus outbreak. The Washington Post website. https://www.washingtonpost. com/religion/2020/03/09/dc-priest-with-coronavirus-leads-prominenthistoric-episcopal-parish/. Published March 9, 2020. Accessed April 3, 2020.

10. Remuzzi A, Remuzzi G. COVID-19 and Italy: what next? The Lancet website. https://www.thelancet.com/journals/lancet/article/PIIS0140-6736(20) 30627-9/fulltext. Published March 13, 2020. Accessed April 3, 2020.

\title{
Artificial intelligence plays an important role in containing public health emergencies
}

\author{
Ningning Tang MD, Guangyi Huang MD, Min Li MD and Fan Xu MD (1) \\ Department of Ophthalmology, People's Hospital of Guangxi Zhuang Autonomous Region, Nanning, Guangxi, China
}

To the Editor-Despite measures taken by the Chinese government to contain coronavirus disease 2019 (COVID-19), its transmission has yet to be halted. Instead, the SARS-Cov-2 virus has demonstrated international seeding leading to a global outbreak. ${ }^{1}$ Although many technical gaps and uncertainties remain in the epidemic management techniques, artificial intelligence (AI) offers the potential for concerned parties to fill these gaps using big data.

Currently, thanks to the support of the Ministry of Industry and Information Technology of China, ${ }^{2}$ research institutions and enterprises across China are actively optimizing AI algorithms and computing power for epidemic control. For example, an automated whole-genome sequencing and analysis platform has been established to promote rapid and accurate virus detection. ${ }^{3}$ Additionally, protein screening, as well as new drugs and vaccine development, have been accelerated thanks to the use of AI. ${ }^{4}$ By applying image-processing algorithms, chest computed tomographic images can be intelligently diagnosed and quantitatively evaluated, and the severity of various types of pneumonia (ranging from local lesions and diffuse lesions to whole-lung involvement) are automatically graded. ${ }^{5}$ Case assessments for COVID-19 using AI systems show acceptable accuracy with high efficiency. ${ }^{5}$ Phonetic interface systems and speech-recognizing robots are frequently employed in healthcare settings to provide intelligent guidance and triage services. ${ }^{6}$ In some communities and public places, these systems can replace healthcare workers in performing some routine tasks, such as temperature measurement, that can be done automatically and accurately through a combination of deep learning, image recognition technology, and infrared imaging sensors. ${ }^{7}$ Smart-dial service can be used employed to conduct phonetic verification with preset conversation content (eg, health status, travel, and contact history), so that residents' health conditions and population mobility can be efficiently investigated. ${ }^{8}$ Facial recognition technology is used to track behavior via real-time processing of video data of close contacts of confirmed and/ or suspected cases. ${ }^{9}$ All in all, the application of AI technology has opened up opportunities for the integration and utilization

Author for correspondence: Fan Xu, E-mail: oph_fan@163.com

Cite this article: Tang N, et al. (2020). Artificial intelligence plays an important role in containing public health emergencies. Infection Control \& Hospital Epidemiology, 41: 869-870, https://doi.org/10.1017/ice.2020.103 of big data across the whole of Chinese society, providing a wealth of information for hospital management, community supervision, and government policy formulation.

Efforts made by a single country remain insufficient. Although the world is currently facing the COVID-19 epidemic, other events, such as a natural disaster followed by a pandemic, might occur in the future as well. In such an event, worldwide data sharing and technology communication will be required to both create and launch a global, public health emergency response plan and to effectively contain the public health emergency. We hope that an open AI-based platform, with epidemiological database integration and comprehensive coverage worldwide, will be established to maximize the benefits of AI and big data. In this way, the nations of the world can cooperate more closely to effectively respond to the common crises that face all of mankind.

Acknowledgments.

Financial support. No financial support was provided relevant to this article.

Conflicts of interest. All authors declare no conflict of interest relevant to this study.

\section{References}

1. Coronavirus disease 2019 (COVID-19) situation report-36. World Health Organization website. https://www.who.int/docs/default-source/coronaviruse/ situation-reports/20200225-sitrep-36-covid-19.pdf?sfvrsn=2791b4e0_2. Published 2020. Accessed February 25, 2020.

2. The proposal of leveraging the power of artificial intelligence to fight against COVID-19. Ministry of Industry and Information Technology of the People's Republic of China website. http://www.miit.gov.cn/n1146290/ n1146402/n1146440/c7664158/content.html. Published 2020. Accessed Feb 4, 2020.

3. Zhejiang Province CDC launches automated whole-genome sequencing and analysis platform. China News website. http://www.chinanews.com/gn/ 2020/02-01/9075431.shtml. Published 2020. Accessed Feb 1, 2020.

4. Zhongshan Nan's team and Alibaba Cloud have reached a cooperation to develop new drugs and vaccine for COVID-19. Xinhuanet website. http:// www.xinhuanet.com/tech/2020-02/14/c_1125574885.htm. Published 2020. Accessed February 14, 2020.

5. Shanghai Public Health Center launched intelligent evaluation system for COVID-19. Netease website. https://3g.163.com/tech/article/F4KKGF6H000 97U80.html. Published 2020. Accessed February 5, 2020. 
6. Iflytek healthcare powered by artificial intelligence. Iflytek website. https:// www.iflytek.com/health. Published 2020. Accessed February 15, 2020.

7. Temperature measurement patrol robot is employed in Guangzhou. Xinhuanet website. http://www.xinhuanet.com/info/2020-02/05/c_138756 758.htm. Published 2020. Accessed February 5, 2020.

8. Hubei Province CDC headquarters establishes "Intelligent Voice Follow-up System." Wuhan broadcasting and TV station website. http:// www.whtv.com.cn/p/23820.html. Published 2020. Accessed February 15,2020

9. The combination of artificial intelligence and face recognition technology for behavior tracking in Shanxi Province. Sina website. http://sx.sina.com.cn/ news/b/2020-02-05/detail-iimxxste8978374.shtml. Published 2020. Accessed February 5, 2020.

\title{
Reducing hand recontamination of healthcare workers during COVID-19
}

\author{
Giorgia Gon $\mathrm{PhD}^{1}$ (1), Stephanie Dancer $\mathrm{PhD}^{2}$, Robert Dreibelbis $\mathrm{PhD}^{3}$, Wendy J. Graham $\mathrm{PhD}^{1}$ and Claire Kilpatrick MSc ${ }^{4}$ \\ ${ }^{1}$ Department of Infectious Disease Epidemiology, London School of Hygiene and Tropical Medicine, London, United Kingdom, ${ }^{2}$ Department of Microbiology, \\ Hairmyres Hospital \& School of Applied Sciences, Edinburgh Napier University, Edinburgh, United Kingdom, ${ }^{3}$ Department of Disease Control, London School of \\ Hygiene and Tropical Medicine, London, United Kingdom and ${ }^{4}$ World Health Organization Consultant, Glasgow, United Kingdom
}

To the Editor - Worldwide, the response to the COVID-19 pandemic requires hand hygiene compliance by everyone, as highlighted in the WHO \#SafeHands campaign and numerous documents. Hand hygiene is particularly critical for frontline healthcare workers (HCWs) who are overstretched and for whom this key routine task must be easy to complete and effective. ${ }^{1}$ However, a neglected aspect of hand hygiene, even in the absence of a global pandemic, is the risk of touching surfaces or objects that could recontaminate hands after hand rubbing or washing, whether gloves are worn or not. Infection prevention is key during this pandemic, and reducing hand recontamination is important to ensuring patient and HCW safety at all times.

Avoiding recontamination is implicit in the WHO Hand Hygiene guidelines for health facilities. ${ }^{2}$ Failure to comply with hand hygiene can result from not washing or rubbing hands at the right time or from subsequent hand or glove recontamination. In a recent study in Tanzania during which 781 hand hygiene indications were observed, approximately half of the times when birth attendants rubbed or washed their hands, they then recontaminated their hands on potentially unclean surfaces before performing an aseptic procedure. ${ }^{3}$ Similar findings come from obstetric wards in Nigeria and Ghana. ${ }^{4,5}$ Recontamination is not only a problem in low-income settings. A US study demonstrated microbiological recontamination of hands at the point of care despite high levels of self-reported hand hygiene compliance. ${ }^{6}$ Reports from the United Kingdom and Australia show that HCWs touch privacy curtains between hand hygiene and touching a patient. ${ }^{7}$

The Tanzanian study also suggested that hand rubbing or washing and glove recontamination are underpinned by different behavioral determinants. ${ }^{8}$ Without targeting these 2 behaviors separately, hand hygiene initiatives during this pandemic may be undermined.

HCWs are able to prioritize patient needs when providing routine care. However, the COVID-19 pandemic has introduced significant uncertainty into the care environment and thus

Author for correspondence: Giorgia Gon, E-mail: giorgia.gon@lshtm.ac.uk

Cite this article: Gon G, et al. (2020). Reducing hand recontamination of healthcare workers during COVID-19. Infection Control \& Hospital Epidemiology, 41: 870-871, https://doi.org/10.1017/ice.2020.111 workflow, including timing of necessary procedures, anticipating and managing patient volumes, and rapidly evolving guidelines on patient management. During this crisis, hand hygiene, along with other infection control activities, may be compromised, not because it is not a priority but rather because staff may be too busy or uncertain on how to implement hand hygiene in this outbreak setting. In their ethnography of infection prevention in Australia, Hor et $\mathrm{al}^{9}$ state that understanding the "boundaries of what is clean" is not straightforward in hospital departments and that HCWs have different perceptions over whether certain surfaces could potentially lead to cross transmission. Recontamination may be an indication that staff fail to understand the definition of the WHO hand hygiene recommendations or how those apply in rapidly changing healthcare settings. ${ }^{3}$

An understanding of surfaces that are safe to touch depend upon assumptions about appropriate cleaning of surfaces, cleaning frequencies, established methods, and sufficient trained cleaning staff. In spite of amazing efforts from all staff, including environmental cleaning staff, standards are not always optimal in the United Kingdom, as in many other countries. ${ }^{10}$ Surface contamination played a plausible role in SARS, MERS, and pandemic influenza transmission in healthcare settings. Emerging evidence suggests that the virus responsible for the current pandemic (SARS-CoV-2) can survive on common surfaces for days, but viral demographics and characteristics have yet to be sufficiently studied. ${ }^{11}$ Recontamination of hands is a consequence and a source of poor surface cleanliness (Fig. 1, Steps 5 and 7).

We call for greater attention to the risk from hand recontamination and the opportunity for its prevention through empowering HCWs and strengthening cleaning of the care environment. For those managing COVID-19 cases, these actions will improve their own and coworkers' safety as well as that of all patients and visitors.

Like so much in the COVID-19 response, behavior change plays a key part. Behavior change needs to be tailored and targeted. Following Michie's principles for behavior change during the COVID-19 pandemic, ${ }^{1}$ we recommend the following:

1. A mental model: Training, monitoring, and feedback should include clear guidance for understanding the "boundaries of what surfaces are clean" with directions on what HCWs can 